\title{
Exposed positive maps: a sufficient condition
}

\author{
Dariusz Chruściński ${ }^{1}$ and Gniewomir Sarbicki ${ }^{1,2}$ \\ ${ }^{1}$ Institute of Physics, Nicolaus Copernicus University, \\ Grudziądzka 5/7, 87-100 Toruń, Poland \\ 2 Stockholms Universitet, Fysikum, S-10691 Stockholm, Sweden
}

\begin{abstract}
Exposed positive maps in matrix algebras define a dense subset of extremal maps. We provide a sufficient condition for a positive map to be exposed. This is an analog of a spanning property which guaranties that a positive map is optimal. We analyze a class of decomposable maps for which this condition is also necessary.
\end{abstract}

\section{Introduction}

Positive maps in $\mathbb{C}^{*}$-algebras play an important role both in mathematics, in connection with the operator theory [1], and in modern quantum physics. Normalized positive maps provide an affine mapping between sets of states of $\mathbb{C}^{*}$-algebras. In recent years positive maps found important application in entanglement theory defining basic tool for detecting quantum entangled states (see e.g. 2] for the recent review).

Let $\mathfrak{U}$ be a unital $\mathbb{C}^{*}$-algebra. A linear map $\Phi: \mathfrak{U} \rightarrow \mathcal{B}(\mathcal{H})$ is positive if $\Phi\left(\mathfrak{U}_{+}\right) \subset \mathcal{B}_{+}(\mathcal{H})$, where $\mathfrak{U}_{+}$denotes positive elements in $\mathfrak{U}$. Denote by $M_{k}(\mathfrak{U})=M_{k}(\mathbb{C}) \otimes \mathfrak{U}$ a space of $k \times k$ matrices with entries from $\mathfrak{U}$. One says that $\Phi$ is $k$-positive if a linear map $\Phi_{k}:=\mathbb{1}_{k} \otimes \Phi: M_{k}(\mathfrak{U}) \rightarrow M_{k}(\mathcal{B}(\mathcal{H}))$ is positive. Finally, $\Phi$ is completely positive if it is $k$-positive for $k=1,2, \ldots$. Due to the Stinespring theorem [3] the structure of completely positive maps is perfectly known: any completely positive map $\Phi$ may be represented in the following form

$$
\Phi(a)=V^{\dagger} \pi(a) V
$$

where $V: \mathcal{H} \rightarrow \mathcal{K}$, and $\pi$ is a representation of $\mathfrak{U}$ in the Hilbert space $\mathcal{K}$. Unfortunately, in spite of the considerable effort, the structure of positive maps is rather poorly understood [4]-23].

Denote by $\mathcal{P}$ a convex cone of positive maps $\Phi: \mathfrak{U} \rightarrow \mathcal{B}(\mathcal{H})$. Note, that a space $\mathcal{L}(\mathfrak{U}, \mathcal{B}(\mathcal{H}))$ of linear maps from $\mathfrak{U}$ to $\mathcal{B}(\mathcal{H})$ is isomorphic to $\mathcal{B}(\mathcal{H}) \otimes \mathfrak{U}$. The natural pairing between these two spaces in defined as follows [13]: taking an orthonormal basis in $\mathcal{H}(m=\operatorname{dim} \mathcal{H}<\infty)$ one identifies $\mathcal{B}(\mathcal{H})$ with $M_{m}(\mathbb{C})$ and defines

$$
\langle X \otimes a, \Phi\rangle:=\operatorname{tr}\left(X^{\mathrm{t}} \Phi(a)\right),
$$

where $X \in M_{m}(\mathbb{C}), a \in \mathfrak{U}$, and $X^{\mathrm{t}}$ denotes transposition of $X$ with respect to a given basis in $\mathcal{H}$. Let $\mathcal{P}^{\circ}$ denote a dual cone [13, 24]

$$
\mathcal{P}^{\circ}=\left\{A \in M_{m}(C) \otimes \mathfrak{U}:\langle A, \Phi\rangle \geq 0, \Phi \in \mathcal{P}\right\} .
$$


Note that the definition of $\mathcal{P}^{\circ}$ may be reformulated as follows

$$
\mathcal{P}^{\circ}=\operatorname{conv}\left\{X \otimes a \in M_{m}(\mathbb{C}) \otimes \mathfrak{U}:\langle X \otimes a, \Phi\rangle \geq 0, \Phi \in \mathcal{P}\right\} .
$$

One finds $\mathcal{P}^{\circ}=M_{m}^{+}(\mathbb{C}) \otimes \mathfrak{U}_{+}$, where $M_{m}^{+}(\mathbb{C})$ denotes positive matrices from $M_{m}(\mathbb{C})$. It shows that $\mathcal{P}^{\circ}$ defines a convex cone of separable elements in $M_{m}(\mathbb{C}) \otimes \mathfrak{U}$.

Recall that a face of $\mathcal{P}$ is a convex subset $F \subset \mathcal{P}$ such that if the convex combination $\Phi=$ $\lambda \Phi_{1}+(1-\lambda) \Phi_{2}$ of $\Phi_{1}, \Phi_{2} \in \mathcal{P}$ belongs to $F$, then both $\Phi_{1}, \Phi_{2} \in F$. If a ray $\{\lambda \Phi: \lambda>0\}$ is a face of $\mathcal{P}$ then it is called an extreme ray, and we say that $\Phi$ generates an extreme ray. For simplicity we call such $\Phi$ an extremal positive map. A face $F$ is exposed if there exists a supporting hyperplane $H$ for a convex cone $\mathcal{P}$ such that $F=H \cap \mathcal{P}$. The property of 'being an exposed face' may be reformulated as follows: A face $F$ of $\mathcal{P}$ is exposed iff there exists $a \in \mathfrak{U}_{+}$and $|h\rangle \in \mathcal{H}$ such that

$$
F=\{\Phi \in \mathcal{P} ; \Phi(a)|h\rangle=0\}
$$

A positive map $\Phi \in \mathcal{P}$ is exposed if it generates 1-dimensional exposed face. Let us denote by $\operatorname{Ext}(\mathcal{P})$ the set of extremal points and $\operatorname{Exp}(\mathcal{P})$ the set of exposed points of $\mathcal{P}$. Due to Straszewicz theorem [24] $\operatorname{Exp}(\mathcal{P})$ is a dense subset of $\operatorname{Ext}(\mathcal{P})$. Thus every extreme map is the limit of some sequence of exposed maps meaning that each entangled state may be detected by some exposed positive map. Hence, a knowledge of exposed maps is crucial for the full characterization of separable/entangled states of bi-partite quantum systems. For recent papers on exposed maps see e.g. [13, 21, 22, 23.

Now, if $F$ is a face of $\mathcal{P}$ then

$$
F^{\prime}=\operatorname{conv}\left\{a \otimes|h\rangle\left\langle h\left|\in \mathcal{P}^{\circ}: \Phi(a)\right| h\right\rangle=0, \Phi \in F\right\} .
$$

defines a face of $\mathcal{P}^{\circ}$ (one calls $F^{\prime}$ a dual face of $F$ ). Actually, $F^{\prime}$ is an exposed face. One proves [13] that a face $F$ is exposed iff $F^{\prime \prime}=F$.

In this paper we analyze linear positive maps $\Phi: \mathcal{B}(\mathcal{K}) \rightarrow \mathcal{B}(\mathcal{H})$, where both $\mathcal{K}$ and $\mathcal{H}$ are finite dimensional Hilbert spaces. We provide a sufficient condition for the map to be exposed. We call it strong spanning property in analogy to well known spanning property which is sufficient for the map to be optimal [26]. Interestingly, this condition is also necessary if $\Phi$ is decomposable and $\operatorname{dim} \mathcal{K}=2$. Finally, we characterize the property of exposedness in terms of entanglement witnesses.

\section{Preliminaries}

Consider a positive map $\Phi: \mathfrak{U} \rightarrow \mathcal{B}(\mathcal{H})$, where $\mathfrak{U}$ is a unital $\mathbb{C}^{*}$-algebra and $\mathcal{B}(\mathcal{H})$ denotes a set of bounded operators on the finite dimensional Hilbert space $\mathcal{H}$.

Proposition 2.1 If $a \in \mathfrak{U}$ is strictly positive, i.e. $a \in \operatorname{int} \mathfrak{U}_{+}$, then $\operatorname{Im} \Phi(b) \subset \operatorname{Im} \Phi(a)$ for all $b \in \mathfrak{U}_{+}$.

Proof: Let us observe that

$$
\operatorname{Ker} \Phi(a) \subset \operatorname{Ker} \Phi(b)
$$


Indeed, suppose that there exists $x \in \mathcal{H}$ such that $x \in \operatorname{Ker} \Phi(a)$ and $x \notin \operatorname{Ker} \Phi(b)$. One has $\langle x|\Phi(b)| x\rangle>0$ and $\langle x|\Phi(a)| x\rangle=0$. Now, since $a \in \operatorname{int} \mathfrak{U}_{+}$there exists $\epsilon>0$ such that an open ball $B(a, \epsilon) \subset \mathfrak{U}_{+}$. It is therefore clear that

$$
a^{\prime}=a-\frac{\epsilon}{2} \frac{u-a}{\|u-a\|}
$$

belongs to $\mathfrak{U}_{+}$. One has

$$
\left\langle x\left|\Phi\left(a^{\prime}\right)\right| x\right\rangle=-\frac{\epsilon}{2\|u-a\|}\langle x|\Phi(u)| x\rangle<0,
$$

which contradicts that $\Phi$ is a positive map. Hence, if $a \in \operatorname{int} \mathfrak{U}_{+}$, then $\operatorname{Ker} \Phi(a) \subset \operatorname{Ker} \Phi(b)$ for any $b \in \mathfrak{U}_{+}$which implies $\operatorname{Im} \Phi(b) \subset \operatorname{Im} \Phi(a)$.

Corollary 2.1 If $a, b \in \operatorname{int} \mathfrak{U}_{+}$, then $\operatorname{Im} \Phi(a)=\operatorname{Im} \Phi(b)$.

Corollary 2.2 In particular for $a \in \mathfrak{U}_{+}\left(a \in \operatorname{int} \mathfrak{U}_{+}\right)$, one has $\operatorname{Im} \Phi(a) \subset \operatorname{Im} \Phi(\mathbb{1})(\operatorname{Im} \Phi(a)=$ $\operatorname{Im} \Phi(\mathbb{1}))$.

Let $A:=\Phi(\mathbb{1})$. If $A>0$, that is, $A$ is of full rank, then one has

$$
\Phi(a)=A^{1 / 2} \widetilde{\Phi}(a) A^{1 / 2},
$$

where $\widetilde{\Phi}(a)=A^{-1 / 2} \Phi(a) A^{-1 / 2}$ is a unital positive map from $\mathfrak{U}$ into $\mathcal{B}(\mathcal{H})$. If $A$ is not strictly positive, that is, $A \in \partial \mathcal{B}_{+}(\mathcal{H})$, then denote by $\mathcal{H}_{\Phi}$ the range of $A$. $A$ is invertible on its image and denote by $\widetilde{A}^{-1}$ the generalized inverse of $A$. Now, one has

$$
\Phi(a)=A^{1 / 2} \widetilde{A}^{-1 / 2} \Phi(a) \widetilde{A}^{-1 / 2} A^{1 / 2} .
$$

Note, that $\operatorname{Im} \Phi(a) \subset \mathcal{H}_{\Phi}$. Following [8] let us introduce the following

Definition 2.1 Consider a positive map $\phi: \mathfrak{U} \rightarrow \mathcal{B}(\mathcal{H})$. A map $\phi^{\prime}: \mathfrak{U} \rightarrow \mathcal{B}\left(\mathcal{H}^{\prime}\right)$ is called an extension of $\phi$ iff $\mathcal{H}^{\prime} \supset \mathcal{H}$ and for any $a \in \mathfrak{U}$

$$
\phi(a)=\mathcal{P} \phi^{\prime}(a) \mathcal{P},
$$

where $\mathcal{P}$ denotes orthogonal projection $\mathcal{H}^{\prime} \rightarrow \mathcal{H}$.

Note that $\mathcal{H}^{\prime}=\mathcal{H} \oplus \mathcal{H}^{\perp}$ and hence for any $\left|h^{\prime}\right\rangle \in \mathcal{H}^{\prime}$ one has $\left|h^{\prime}\right\rangle=|h\rangle \oplus\left|h^{\perp}\right\rangle$, where $|h\rangle=\mathcal{P}\left|h^{\prime}\right\rangle$ which implies $\phi(a)|h\rangle=\mathcal{P} \phi^{\prime}(a)|h\rangle$, and an extension $\Phi^{\prime}$ is trivial if

$$
\phi^{\prime}(a)|h\rangle=\phi(a)|h\rangle
$$

for all $a \in \mathfrak{U}$ and $|h\rangle \in \mathcal{H}$. According to this definition a positive map $\widetilde{\Phi}(a):=\widetilde{A}^{-1 / 2} \Phi(a) \widetilde{A}^{-1 / 2}$ is a trivial extension of the unital map $\Phi_{1}: \mathfrak{U} \rightarrow \mathcal{B}\left(\mathcal{H}_{\Phi}\right)$

$$
\Phi_{1}=\mathcal{P}_{\Phi} \widetilde{\Phi} \mathcal{P}_{\Phi}
$$

where $\mathcal{P}_{\Phi}$ is a projector $\mathcal{H} \rightarrow \mathcal{H}_{\Phi}$. This way we proved the following 
Proposition 2.2 Any linear positive map $\Phi: \mathfrak{U} \rightarrow \mathcal{B}(\mathcal{H})$ can be written as follows

$$
\Phi(a)=V^{\dagger} \Phi_{1}(a) V
$$

where $V: \mathcal{H} \rightarrow \mathcal{H}_{\Phi}$ and $\Phi_{1}: \mathfrak{U} \rightarrow \mathcal{B}\left(\mathcal{H}_{\Phi}\right)$ is unital.

Let us recall

Definition 2.2 A linear map $\Phi$ is irreducible if $[\Phi(a), X]=0$ for all $a \in \mathfrak{U}$ implies that $X=\lambda \mathbb{I}_{\mathcal{H}}$. $\Phi$ is irreducible on its image if $[\Phi(a), X]=0$ for all $a \in \mathfrak{U}$ implies that $\mathcal{P}_{\Phi} X \mathcal{P}_{\Phi}=\lambda \mathbb{I}_{\mathcal{H}_{\Phi}}$.

Remark 2.1 Note, that one may restrict oneself to self-adjoint elements $\mathfrak{U}_{\mathrm{sa}}$ only. Indeed, suppose that $\Phi$ is irreducible and $[\Phi(a), X]=0$ for all $a \in \mathfrak{U}_{\mathrm{sa}}$. Any element $x \in \mathfrak{U}$ may be decomposed as $x=x_{1}+i x_{2}$, with $x_{1}, x_{2} \in \mathfrak{U}_{\text {sa }}$. One has

$$
[\Phi(x), X]=\left[\Phi\left(x_{1}\right), X\right]+i\left[\Phi\left(x_{2}\right), X\right]=0,
$$

and irreducibility of $\Phi$ implies therefore $X=\lambda \mathbb{I}_{\mathcal{H}_{\Phi}}$.

Proposition 2.3 Let a positive map $\Phi$ be irreducible. If $X \Phi(a)=\Phi(a) X^{\dagger}$ for all $a \in \mathfrak{U}$, then $X=\lambda \mathbb{I}_{\mathcal{H}}$.

Proof: Irreducibility implies that $A=\Phi(\mathbb{1})>0$ and hence

$$
\Phi(a)=A^{1 / 2} \Phi_{1}(a) A^{1 / 2},
$$

where $\Phi_{1}$ is unital. One has

$$
X A^{1 / 2} \Phi_{1}(a) A^{1 / 2}=A^{1 / 2} \Phi_{1}(a) A^{1 / 2} X^{\dagger},
$$

and hence

$$
Y \Phi_{1}(a)=\Phi_{1}(a) Y^{\dagger}
$$

with $Y=A^{-1 / 2} X A^{1 / 2}$. Using $\Phi_{1}(\mathbb{1})=\mathbb{I}_{\mathcal{H}}$ one finds $Y^{\dagger}=Y$. Let us observe that $\Phi_{1}$ is irreducible as well and hence $Y=\lambda \mathbb{I}_{\mathcal{H}}$ which implies $X=\lambda \mathbb{I}_{\mathcal{H}}$.

\section{Exposed maps - sufficient condition}

In this section we formulate a sufficient condition for a map $\Phi: \mathcal{B}(\mathcal{K}) \longrightarrow \mathcal{B}(\mathcal{H})$ to be exposed. Recall that a linear operator $W \in \mathcal{B}(\mathcal{K} \otimes \mathcal{H})$ is block-positive iff $\langle x \otimes y|W| x \otimes y\rangle \geq 0$ for all product vectors $|x \otimes y\rangle \in \mathcal{K} \otimes \mathcal{H}$. Now, due to the Choi-Jamiołkowski isomorphism, $W$ is block-positive iff there exists a positive map $\Phi: \mathcal{B}(\mathcal{K}) \rightarrow \mathcal{B}(\mathcal{H})$ such that

$$
W=\left(\mathbb{1}_{\mathcal{K}} \otimes \Phi\right) P_{\mathcal{K}}^{+},
$$

where $\mathbb{1}_{\mathcal{K}}$ is an identity map in $\mathcal{B}(\mathcal{K})$, and $P_{\mathcal{K}}^{+}$is a maximally entangled state in $\mathcal{K} \otimes \mathcal{K}$. Any block-positive but not positive $W$ is called an entanglement witness. It is therefore clear that any property of a map $\Phi$ may be formulated in terms of $W$ and vice versa. Now, let us define

$$
P_{W}=\{x \otimes y:\langle x \otimes y|W| x \otimes y\rangle=0\} .
$$


Note, that

$$
\langle x \otimes y|W| x \otimes y\rangle=\langle y|\Phi(|\bar{x}\rangle\langle\bar{x}|)| y\rangle,
$$

and hence one may equivalently introduce $P_{\Phi} \equiv P_{W}=\{x \otimes y: \Phi(|\bar{x}\rangle\langle\bar{x}|)|y\rangle=0\}$. One says that $\Phi$ has spanning property iff $\operatorname{span}_{\mathbb{C}} P_{\Phi}=\mathcal{K} \otimes \mathcal{H}$. Denoting $d_{\mathcal{K}}=\operatorname{dim} \mathcal{K}$ and $d_{\mathcal{H}}=\operatorname{dim} \mathcal{H}$, one proves [26]

Theorem 3.1 If a positive map $\Phi$ satisfies spanning property, then it is optimal.

In analogy we have the following

Theorem 3.2 Let $\Phi: \mathcal{B}(\mathcal{K}) \longrightarrow \mathcal{B}(\mathcal{H})$ be a positive map irreducible on its image and

$$
N_{\Phi}=\operatorname{span}_{\mathbb{C}}\left\{a \otimes|h\rangle \in \mathcal{B}_{+}(\mathcal{K}) \otimes \mathcal{H}: \Phi(a)|h\rangle=0\right\} .
$$

If the subspace $N_{\Phi} \subset \mathcal{B}(\mathcal{K}) \otimes \mathcal{H}$ satisfies

$$
\operatorname{dim} N_{\Phi}=d_{\mathcal{K}}^{2} d_{\mathcal{H}}-\operatorname{rank} \Phi\left(\mathbb{I}_{\mathcal{K}}\right)
$$

then $\Phi$ is exposed.

Proof: The idea of the proof comes from [8] (see Theorem 3.3). Consider a map 25]

$$
\tilde{\Phi}: \mathcal{B}(\mathcal{K}) \otimes \mathcal{H} \longrightarrow \mathcal{H}
$$

defined by

$$
\tilde{\Phi}(a \otimes|h\rangle):=\Phi(a)|h\rangle .
$$

Note, that $\operatorname{dim}(\operatorname{Im} \tilde{\Phi})=\operatorname{rank} \Phi\left(\mathbb{I}_{\mathcal{K}}\right)$ and hence $N_{\Phi}$ defines the kernel of $\tilde{\Phi}$. To show that $\Phi$ is exposed let us introduce a linear functional $f$ on the space of positive maps $\mathcal{B}(\mathcal{K}) \rightarrow \mathcal{B}(\mathcal{H})$ defined as follows

$$
f(\Psi)=\sum_{i=1}^{d_{N}}\left\langle h_{i}\left|\Psi\left(a_{i}\right)\right| h_{i}\right\rangle,
$$

where $d_{N}$ vectors $a_{i} \otimes\left|h_{i}\right\rangle$ span $N_{\Phi}$. Note that $f(\Psi) \geq 0$ for all positive maps $\Psi$ and $f(\Phi)=0$. As a result $f$ defines a supporting hyperplane to the cone of positive maps $\mathcal{B}(\mathcal{K}) \rightarrow \mathcal{B}(\mathcal{H})$ passing through a map $\Phi$. Note that $\Phi$ is exposed iff $f(\Psi)=0$ implies $\Psi=\lambda \Phi$, with $\lambda$ being a positive number. Let us observe that $f(\Psi)=0$ if and only if $\tilde{\Psi}\left(a_{i} \otimes\left|h_{i}\right\rangle\right)=\Psi\left(a_{i}\right)\left|h_{i}\right\rangle=0$, for all $i=1, \ldots, d_{N}$, and hence the kernel of $\tilde{\Psi}$ contains $N_{\Phi}$. To complete the proof we use the following

Lemma 3.1 Consider two linear operators $A, B: V \rightarrow W$, where $V$ and $W$ are finite dimensional vector spaces over $\mathbb{C}$. If $\operatorname{ker} A \supset \operatorname{ker} B$, then there exists $X: W \rightarrow W$ such that $A=X B$ and $\operatorname{rank} X=\operatorname{rank} A$.

Proof: let

$$
A=U_{A} \Sigma_{A} V_{A}^{\dagger}, \quad B=U_{B} \Sigma_{B} V_{B}^{\dagger}
$$


denote the corresponding singular value decompositions of $A$ and $B$. Let $\left\{v_{\alpha}(A)\right\},\left\{w_{\alpha}(A)\right\}$, $\left\{v_{\alpha}(B)\right\}$ and $\left\{w_{\alpha}(B)\right\}$ denote the orthonormal basis made from columns of $V_{A}, U_{A}, V_{B}, U_{B}$ respectively. One has

$$
\Sigma_{A}=\sum_{\alpha=1}^{r_{A}} \sigma_{\alpha}(A)\left|w_{\alpha}(A)\right\rangle\left\langle v_{\alpha}(A)\left|, \quad \Sigma_{B}=\sum_{\alpha=1}^{r_{B}} \sigma_{\alpha}(B)\right| w_{\alpha}(B)\right\rangle\left\langle v_{\alpha}(B)\right|,
$$

where $\sigma_{\alpha}(A)$ and $\sigma_{\alpha}(B)$ are strictly positive singular values of $A$ and $B$, respectively. Note, that condition $\operatorname{ker} A \supset \operatorname{ker} B$, is equivalent to $r_{B} \geq r_{A}$. One finds $A=X B$, where

$$
X=A V_{B}^{\dagger} \widetilde{\Sigma}_{B} U_{B}^{\dagger}
$$

with

$$
\widetilde{\Sigma}_{B}=\sum_{\alpha=1}^{r_{B}} \sigma_{\alpha}(B)^{-1}\left|w_{\alpha}\right\rangle\left\langle v_{\alpha}\right|
$$

Indeed, one has

$$
X B=\left(A V_{B} \widetilde{\Sigma}_{B} U_{B}^{\dagger}\right)\left(U_{B} \Sigma_{B} V_{B}^{\dagger}\right)=A V_{B} \widetilde{\Sigma}_{B} \Sigma_{B} V_{B}^{\dagger}=A \sum_{\alpha=1}^{r_{B}}\left|v_{\alpha}(B)\right\rangle\left\langle v_{\alpha}(B)\right| .
$$

Now, since $\operatorname{ker} A \supset \operatorname{ker} B$, one has

$$
A \sum_{\alpha=1}^{r_{B}}\left|v_{\alpha}(B)\right\rangle\left\langle v_{\alpha}(B)\right|=A
$$

which ends the proof.

One has, therefore, $\tilde{\Psi}=X \tilde{\Phi}$, for some operator $X$ acting on the image of $\tilde{\Phi}$, meaning that

$$
\Psi(a)|h\rangle=X \Phi(a)|h\rangle,
$$

for all $a \in \mathcal{B}(\mathcal{K})$ and $|h\rangle \in \mathcal{H}$. Note that for any $a \in \mathcal{B}_{\text {sa }}(\mathcal{K})$ one has $\Psi(a)=\Psi(a)^{\dagger}$ and hence

$$
X \Phi(a)=\Phi(a) X^{\dagger} .
$$

Proposition 2.3 implies, therefore, that $X \sim \mathbb{I}$ on the image of $\Phi$. Hence $\Psi=\lambda \Phi$ with $\lambda>0$ due to the fact that both $\Phi$ and $\Psi$ are positive maps.

Corollary 3.1 Let $\Phi: \mathcal{B}(\mathcal{K}) \longrightarrow \mathcal{B}(\mathcal{H})$ be a positive, unital irreducible map. If

$$
\operatorname{dim} N_{\Phi}=\left(d_{\mathcal{K}}^{2}-1\right) d_{\mathcal{H}},
$$

then $\Phi$ is exposed.

We propose to call (18) strong spanning property in analogy to spanning poperty

$$
\operatorname{dim} \operatorname{span}_{\mathbb{C}}\{|x\rangle \otimes|h\rangle \in \mathcal{K} \otimes \mathcal{H}: \Phi(|\bar{x}\rangle\langle\bar{x}|)|h\rangle=0\}=d_{\mathcal{K}} d_{\mathcal{H}},
$$

which is sufficient for optimality. 


\section{A class of exposed decomposable maps $\mathcal{B}\left(\mathbb{C}^{2}\right) \longrightarrow \mathcal{B}\left(\mathbb{C}^{m}\right)$}

In this section we provide a class of positive exposed maps for which strong spanning property (18) is also necessary.

Theorem 4.1 Let $\Phi: \mathcal{B}\left(\mathbb{C}^{2}\right) \rightarrow \mathcal{B}\left(\mathbb{C}^{m}\right)$ be a decomposable positive but not completely positive map. Then the following conditions are equivalent:

1. $\Phi$ is exposed.

2. $\Phi(\rho)=V^{\dagger} \rho^{t} V$, where $V: \mathbb{C}^{n} \rightarrow \mathbb{C}^{2}$ is a linear map of rank two.

3. There are $4 m-2$ linearly independent vectors in the set $\left\{a \otimes|h\rangle \in \mathcal{B}_{+}\left(\mathbb{C}^{2}\right) \otimes \mathbb{C}^{m}: \Phi(a)|h\rangle=\right.$ $0\}$.

Proof: $(1 \Rightarrow 2)$ Any exposed map is extremal and hence being a decomposable map $\Phi$ is given by $\Phi(a)=V^{\dagger} a V$ or $\Phi(a)=V^{\dagger} a^{\mathrm{t}} V$. The former is evidently $\mathrm{CP}$ and the latter in not CP iff $\operatorname{rank}(V)=2$.

$(2 \Rightarrow 3)$ Note, that using linear transformation one can transform $V$ to the following form $V=\sum_{i=1}^{2}\left|e_{i}\right\rangle\left\langle f_{i}\right|$, where $\left\{e_{i}\right\}_{i=1}^{2},\left\{f_{j}\right\}_{j=1}^{m}$ are orthonormal bases in $\mathbb{C}^{2}$ and $\mathbb{C}^{m}$, respectively. One finds $4(m-2)$ independent vectors taking $a \in \mathcal{B}_{+}\left(\mathbb{C}^{2}\right)$ arbitrary and $|h\rangle=\sum_{j=3}^{m} h_{j} f_{j}$. Now, we look for the remaining vectors $a \otimes|h\rangle$, with $|h\rangle=h_{1} f_{1}+h_{2} f_{2}$. It is clear that it is enough to consider $a \in \mathcal{B}_{+}\left(\mathbb{C}^{n}\right)$ being rank-1 projector, i.e. $a=|x\rangle\langle x|$. One has

$$
\Phi(|x\rangle\langle x|)|h\rangle=V^{\dagger}|\bar{x}\rangle\langle\bar{x}|V| h\rangle=\left(\sum_{i=1}^{2} x_{i} h_{i}\right) \sum_{j=1}^{2} \bar{x}_{j}\left|f_{j}\right\rangle .
$$

Note that $\Phi(|x\rangle\langle x|)|h\rangle=0$ for $|x\rangle \neq 0$ if and only if $\sum_{i=1}^{2} x_{i} h_{i}=0$, and hence (up to trivial scaling) $x_{1}=h_{2}$ and $x_{2}=-h_{1}$. The family of vectors $|x\rangle\langle x|\otimes| h\rangle \in \mathcal{B}\left(\mathbb{C}^{2}\right) \otimes \mathbb{C}^{m}$ is linearly independent iff the corresponding vectors $|\bar{x}\rangle \otimes|x\rangle \otimes|h\rangle$ are linearly independent in $\mathbb{C}^{2} \otimes \mathbb{C}^{2} \otimes \mathbb{C}^{m}$. Note that coordinates of $\bar{x} \otimes x \otimes h$ are polynomial functions of $h_{k}$ and $\bar{h}_{k}$, namely:

$$
h_{1} h_{2} \bar{h}_{2}, h_{1} h_{2} \bar{h}_{1}, h_{1}^{2} \bar{h}_{2}, h_{1} h_{2} \bar{h}_{2}, h_{2}^{2} \bar{h}_{2}, h_{2}^{2} \bar{h}_{1}, h_{2} h_{1} \bar{h}_{2}, h_{2}^{2} \bar{h}_{2} \text {. }
$$

Note, that 6 of them are (functionally) linearly independent and hence one has 6 additional vectors $a \otimes|h\rangle$. Altogether, there are $4(m-2)+6=4 m-2$ linearly independent vectors.

$(3 \Rightarrow 1)$ Follows from Theorem 3.2 .

A similar problem was analyzed in [27] in the context of optimal decomposable maps. Recall that $\Phi$ is decomposable if $\Phi=\Phi_{1}+\Phi_{2} \circ$ t, where $\Phi_{1}$ and $\Phi_{2}$ are completely positive. Equivalently, the corresponding entanglement witness $W$ is decomposable if $W=Q_{1}+\left(\mathbb{1}_{\mathcal{H}} \otimes \mathrm{t}\right) Q_{2}$, where $Q_{1}, Q_{2} \in$ $\mathcal{B}_{+}(\mathcal{H} \otimes \mathcal{K})$. Let us recall that $S \subset \mathcal{H} \otimes \mathcal{K}$ is a completely entangled subspace (CES) iff there is no nonzero product vectors in $S$. The authors of [27] proved the following

Theorem 4.2 Let $\Phi: \mathcal{B}\left(\mathbb{C}^{2}\right) \longrightarrow \mathcal{B}\left(\mathbb{C}^{m}\right)$ be a positive decomposable map. The following conditions are equivalent

1. $\Phi$ is optimal, 
2. $\Phi(a)=\operatorname{Tr}_{\mathbb{C}^{2}}\left(W a^{\mathrm{t}} \otimes \mathbb{I}_{m}\right)$, where $W=\left(\mathbb{1}_{2} \otimes \mathrm{t}\right) Q$ and $Q \geq 0$ is supported on a CES,

3. $P_{\Phi}$ spans $\mathbb{C}^{2} \otimes \mathbb{C}^{m}$.

Note, that we replaced optimality by exposedness, an arbitrary CES by a 1-dimensional CES supporting a positive operator

$$
Q=\sum_{i, j=1}^{2}|i\rangle\left\langle j\left|\otimes V^{\mathrm{t}}\right| i\right\rangle\langle j| \bar{V},
$$

with $\operatorname{rank}(V)=2$ (clearly, if $\operatorname{rank}(V)=1$, then $Q$ is no longer supported on a CES). Finally, we replaced weak spanning property

$$
\operatorname{dim} \operatorname{span}_{\mathbb{C}}\{|\bar{x}\rangle \otimes|h\rangle: \Phi(|x\rangle\langle x|)|h\rangle=0\}=2 m,
$$

by much stronger property (strong spanning)

$$
\operatorname{rank} \Phi\left(\mathbb{I}_{2}\right)+\operatorname{dim} \operatorname{span}_{\mathbb{C}}\{|\bar{x}\rangle \otimes|x\rangle \otimes|h\rangle: \Phi(|x\rangle\langle x|)|h\rangle=0\}=4 m .
$$

\section{$5 \quad$ A class of exposed decomposable maps $\mathcal{B}\left(\mathbb{C}^{n}\right) \longrightarrow \mathcal{B}\left(\mathbb{C}^{m}\right)$}

It was already shown by Marciniak [21] that all extremal decomposable maps $\mathcal{B}\left(\mathbb{C}^{n}\right) \longrightarrow \mathcal{B}\left(\mathbb{C}^{m}\right)$ are exposed, i.e. maps of the form $\Phi(a)=V^{\dagger} a V$ and $\Phi(a)=V^{\dagger} a^{\mathrm{t}} V$ are exposed. Now we show that being exposed these maps in general do not satisfy the strong spanning property (18).

Proposition 5.1 Consider a positive decomposable map $\Phi: \mathcal{B}\left(\mathbb{C}^{n}\right) \rightarrow \mathcal{B}\left(\mathbb{C}^{m}\right)$ defined by $\Phi(a)=$ $V^{\dagger} a^{\mathrm{t}} V$. One has

$$
\operatorname{dim} N_{\Phi}=\left\{\begin{array}{cc}
m\left(n^{2}-1\right) & , \operatorname{rank}(V)>1 \\
m n^{2}-(2 m-1) & , \operatorname{rank}(V)=1
\end{array}\right.
$$

Proof: it is clear that it is enough to consider $a \in \mathcal{B}\left(\mathbb{C}^{n}\right)_{+}$being rank-1 projector, i.e. $a=$ $|x\rangle\langle x|$. Note, that using a linear transformation one can transform $V$ to the following form $V=$ $\sum_{i=1}^{r}\left|e_{i}\right\rangle\left\langle f_{i}\right|$, where $\left\{e_{i}\right\}_{i=1}^{n},\left\{f_{j}\right\}_{j=1}^{m}$ are orthonormal bases in $\mathbb{C}^{n}$ and $\mathbb{C}^{m}$, respectively.

Let $|\tilde{x}\rangle$ and $|\tilde{h}\rangle$ be vectors in $\mathbb{C}^{r}$ built from the first $r$ coordinates of $|x\rangle$ and $|h\rangle$, respectively. For a given vector $|x\rangle$, the orthogonal complement of $|\tilde{x}\rangle$ is spanned by $r-1$ vectors

$$
v_{2}=\left|-x_{2}, x_{1}, 0, \ldots, 0\right\rangle, \quad v_{3}=\left|-x_{2}, 0, x_{1}, 0, \ldots, 0\right\rangle, \ldots, v_{r}=\left|-x_{r}, \ldots, x_{1}\right\rangle .
$$

The general vector $|h\rangle$ orthogonal to $|x\rangle$ is then of the form $\sum_{i=2}^{r} \alpha_{i}\left|v_{i}\right\rangle \oplus\left|\hat{h}_{i}\right\rangle$ (where $\sum_{i=2}^{r} \alpha_{i}\left|\hat{h}_{i}\right\rangle=$ $\left.\left|h_{r+1}, \ldots, h_{m}\right\rangle\right)$. Observe, that $\left|h_{r+1}, \ldots, h_{m}\right\rangle$ can be arbitrary. Now, a general vector $|h\rangle$ which is orthogonal to $|x\rangle$ is a linear combination of vectors from $r-1$ subspaces:

$$
\begin{aligned}
& H_{2}(x)=\operatorname{span}_{\mathbb{C}}\left\{\left|-x_{2}, x_{1}, 0, \ldots, 0\right\rangle\right\} \oplus \mathbb{C}^{m-r}, \\
& H_{3}(x)=\operatorname{span}_{\mathbb{C}}\left\{\left|-x_{3}, 0, x_{1}, 0, \ldots, 0\right\rangle\right\} \oplus \mathbb{C}^{m-r}, \\
& \vdots \\
& H_{r}(x)=\operatorname{span}_{\mathbb{C}}\left\{\left|-x_{r}, 0, \ldots, 0, x_{1}\right\rangle\right\} \oplus \mathbb{C}^{m-r} .
\end{aligned}
$$


Consider the subspace $W_{2} \subset \mathbb{C}^{n} \otimes \mathbb{C}^{n} \otimes \mathbb{C}^{m} \approx \mathcal{B}\left(\mathbb{C}^{n}\right) \otimes \mathbb{C}^{m}$ spanned by the vectors $|\bar{x}\rangle \otimes|x\rangle \otimes|h\rangle$, where $|h\rangle \in H_{2}(x)$, that is, $|\bar{x}\rangle \otimes|y\rangle$, where

$$
|y\rangle=\left|x_{1}, \ldots, x_{r}, x_{r+1}, \ldots, x_{n}\right\rangle \otimes\left|-x_{2}, x_{1}, 0, \ldots, 0, h_{r+1}, \ldots, h_{m}\right\rangle=\sum_{i, j} y_{i j} e_{i} \otimes f_{j} .
$$

The coordinates of $|y\rangle$ are monomials of degree 2 in variables $\left\{x_{1}, \ldots, x_{n}, h_{r+1}, \ldots, h_{m}\right\}$. Note that $|y\rangle$ has in general $(2+m-r) \times n$ non-zero coordinates, which satisfy one linear condition $y_{11}+y_{22}=0$. Hence $\operatorname{dim} W_{2}=n(n[m-r+2]-1)$. Using the same argument one shows that $\operatorname{dim} W_{2}=\ldots=\operatorname{dim} W_{r}$. It is easy to show that

$$
W_{i} \cap W_{j}=W_{2} \cap \cdots \cap W_{r},
$$

for each pair $i \neq j$. Moreover, the constructions of $H_{i}(x)$ imply

$$
W_{2} \cap \cdots \cap W_{r}=\mathbb{C}^{n} \otimes\left(\operatorname{span}_{\mathbb{C}}\left\{e_{2}, \ldots, e_{n}\right\} \otimes f_{1} \oplus \mathbb{C}^{n} \otimes \operatorname{span}_{\mathbb{C}}\left\{f_{r+1}, \ldots, f_{m}\right\}\right),
$$

and hence its dimension equals $n(n-1+[m-r] n)$. Let $W=\operatorname{span}_{\mathbb{C}}\left(W_{2} \cup \ldots \cup W_{r}\right)$. One finds

$$
\begin{aligned}
\operatorname{dim} W & =\sum_{i=2}^{r} \operatorname{dim} W_{i}-(r-2) \cdot \operatorname{dim}\left(W_{2} \cap \cdots \cap W_{r}\right) \\
& =(r-1) n((m-r+2) n-1)-(r-2) n(n-1+(m-r) n)=n^{2} m-n .
\end{aligned}
$$

Note that if $r=1$, one consider vectors $|\bar{x}\rangle \otimes|x\rangle \otimes|h\rangle$ such that $x_{1} h_{1}=0$. Vectors with $x_{1}=0$ form a $(n-1)^{2} m$ dimensional subspace. Vectors with $h_{1}=0$ form a $n^{2}(m-1)$ dimensional subspace. The intersection of these subspaces is $(n-1)^{2}(m-1)$ dimensional. Finally, one gets $(n-1)^{2} m+n^{2}(m-1)-(n-1)^{2}(m-1)=n^{2} m-(2 n-1)$ linearly independent vectors.

It is therefore clear that the strong spanning property

$$
\operatorname{rank} \Phi\left(\mathbb{I}_{n}\right)+\operatorname{dim} \operatorname{span}_{\mathbb{C}}\{|\bar{x}\rangle \otimes|x\rangle \otimes|h\rangle: \Phi(|x\rangle\langle x|)|h\rangle=0\}=m n^{2},
$$

supplemented by irreducibility provides only a sufficient condition for exposedness in the same way as weak spanning property

$$
\operatorname{dim} \operatorname{span}_{\mathbb{C}}\{|\bar{x}\rangle \otimes|h\rangle: \Phi(|x\rangle\langle x|)|h\rangle=0\}=n m,
$$

provides only a sufficient condition for optimality. Note, that $\Phi(a)=V^{\dagger} a^{\mathrm{t}} V$ has a strong spanning property iff $\operatorname{rank}(V)=n$. However, $\Phi$ is exposed for any $V$ [21].

\section{Conclusions}

We have provided a sufficient condition for exposedness - strong spanning property (18). It was shown that in the class of decomposable maps $\mathcal{B}\left(\mathbb{C}^{n}\right) \longrightarrow \mathcal{B}\left(\mathbb{C}^{m}\right)$ this condition is also necessary if $n=2$. This result provides an analog of the result of [27] in the context of optimal maps/witnesses. One calls a block-positive operator $W \in \mathcal{B}\left(\mathbb{C}^{n} \otimes \mathbb{C}^{m}\right)$ irreducible iff $W$ cannot be written as $W_{1} \oplus W_{2}$, where $W_{1}$ and $W_{2}$ are block-positive. One has the following 
Proposition 6.1 Let $W \in \mathcal{B}\left(\mathbb{C}^{n} \otimes \mathbb{C}^{m}\right)$ be a block-positive irreducible operator. If

$$
\operatorname{dim}\left(\operatorname{Im} \operatorname{Tr}_{\mathbb{C}^{n}} W\right)+\operatorname{dim}\left\{a \otimes h: \operatorname{Tr}_{\mathbb{C}^{n}}\left[W a^{\mathrm{t}} \otimes \mathbb{I}_{m}\right]|h\rangle=0\right\}=n^{2} m
$$

then $W$ is exposed.

If $n=2$, then one proves the following

Proposition 6.2 Let $W \in \mathcal{B}\left(\mathbb{C}^{2} \otimes \mathbb{C}^{m}\right)$ be a block-positive but not positive decomposable operator (i.e. decomposable entanglement witness). The following conditions are equivalent

1. $W$ is exposed,

2. $W=\left(\mathbb{1}_{2} \otimes \mathrm{t}\right) Q$, and $Q$ is Schmidt rank 2 projector,

3. There are $3 m$ linearly independent vectors $|\bar{x}\rangle\langle\bar{x}|\otimes| h\rangle \in \mathcal{B}_{+}\left(\mathbb{C}^{2} \otimes \mathbb{C}^{n}\right)$ such that

$$
\langle x \otimes h|W| x \otimes h\rangle=0
$$

In the forthcoming paper we use the strong spanning property to analyze exposed positive indecomposable maps.

\section{Acknowledgments}

It's a pleasure to thank Professor Woronowicz for interesting discussions about exposed and nonextendible maps and Professor Kye for inspiring discussion about facial structure of the cone of positive maps. G.S. was partially supported by research fellowship within project Enhancing Educational Potential of Nicolaus Copernicus University in the Disciplines of Mathematical and Natural Sciences (project no. POKL.04.01.01-00-081/10.)

\section{References}

[1] V. Paulsen, Completely Bounded Maps and Operator Algebras, Cambridge University Press, 2003.

[2] R. Horodecki, P. Horodecki, M. Horodecki and K. Horodecki, Rev. Mod. Phys. 81, 865 (2009).

[3] W.F. Stinespring, Proc. Amer. Math. Soc. 6, 211 (1955).

[4] E. Størmer, in Lecture Notes in Physics 29, Springer Verlag, Berlin, 1974, pp. 85-106; Acta Math. 110, 233 (1963); Proc. Am. Math. Soc. 86, 402 (1982).

[5] M.-D. Choi, Linear Alg. Appl. 12, 95 (1975); J. Operator Theory, 4, 271 (1980).

[6] M.-D. Choi and T.-T. Lam, Math. Ann. 231, 1 (1977).

[7] S. L. Woronowicz, Rep. Math. Phys. 10, 165 (1976). 
[8] S. L. Woronowicz, Comm. Math. Phys. 51, 243 (1976).

[9] A. G. Robertson, J. London Math. Soc. (2) 32, 133 (1985).

[10] K. Tanahashi and J. Tomiyama, Canad. Math. Bull. 31, 308 (1988).

[11] H. Osaka, Publ. Res. Inst. Math. Sci. 28, 747 (1992).

[12] S.-H. Kye, Canad. Math. Bull. 39, 74 (1996); Linear Alg. Appl. 362, 57 (2003); Positivity, 9, 63 (2005).

[13] M.-H. Eom and S.-H. Kye, Math. Scand. 86, 130 (2000).

[14] K.-C. Ha, S.-H. Kye and Y. S. Park, Phys. Lett. A 313, 163 (2003).

[15] K.-C. Ha and S.-H. Kye, Phys. Lett. A 325, 315 (2004); Phys. Rev. A 84, 024302 (2011).

[16] G. Sarbicki, J. Phys. A: Math. Theor. 41, 375303 (2008).

[17] D. Chruściński and A. Kossakowski, J. Phys. A: Math. Theor. 41, (2008), 145301; J. Phys. A: Math. Theor. 41, 215201 (2008); Phys. Lett. A 3732301 (2009).

[18] D. Chruściński and A. Kossakowski, Comm. Math. Phys. 290, 1051 (2009).

[19] D. Chruściński and J. Pytel, J. Phys. A: Math. Theor. 44, 165304 (2011).

[20] D. A. Yopp and R. D. Hill, Linear and Multilinear Algebra, 53, 167 (2005).

[21] M. Marciniak, Rank properties of exposed positive maps, arXiv:1103.3497.

[22] W. A. Majewski, On the structure of positive maps; finite dimensional case, arXiv:1005.3949.

[23] K-C. Ha and S-H. Kye, Open Sys. Inf. Dyn. 18, 323 (2011) .

[24] R. T. Rockafellar, Convex Analysis, Princeton University Press, 1970.

[25] S.L. Woronowicz, private communication

[26] M. Lewenstein, B. Kraus, J. I. Cirac, and P. Horodecki, Phys. Rev. A 62, 052310 (2000).

[27] R. Augusiak, J. Tura, and M. Lewenstein, J. Phys. A: Math. Theor. 44, 212001 (2011). 